IMMUNOMETABOLISM

\section{Getting enough energy for immunity}

Mounting an immune response against pathogens is an energetically demanding process. Because of a sickness-induced reduction in food intake, fuelling this response requires mobilization of stored fuels. Now, a new study by Ajay Chawla and colleagues shows that mice enter an energy-conserving state in response to infection that prioritizes immunity over maintenance of normal body temperature (homeothermy), resulting in hypometabolism and hypothermia.

The authors investigated the competition for energy between immunity and physiological energy-consuming processes such as maintenance of a basal metabolic rate (BMR), physical activity and homeothermy. To study this, mice were treated with lipopolysaccharide (LPS) to activate an innate immune response and housed at different ambient temperatures. When housed at $30^{\circ} \mathrm{C}$ (thermoneutrality), mice do not need to expend any additional energy to maintain their normal body temperature, whereas when housed at $22^{\circ} \mathrm{C}$, mice depend on adaptive thermogenesis to maintain their core temperature. In both groups, LPS administration induced energy conservation (measured as a reduction in oxygen consumption), but this was achieved through different means. In mice housed at

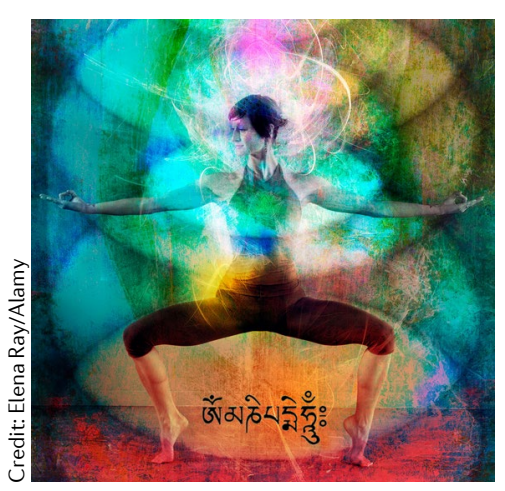

$30^{\circ} \mathrm{C}$, energy saving due to lowered physical activity was sufficient to fuel immune activation and maintain a normal body temperature. However, mice housed at $22^{\circ} \mathrm{C}$ required a combination of reduced physical activity, a lower BMR and decreased adaptive thermogenesis to mount a comparable immune response to LPS. This suggests that the metabolic cost of innate immune activation induces a trade-off between different energy-consuming processes.

The authors also explored how these competing metabolic demands are met during immune activation. Because LPS-induced sickness behaviours decrease food intake, immune activation resulted in a substrate shift from ingested carbohydrates to ketone bodies (indicative of stored fuel source usage) in both groups of mice. The observation that mice lost weight primarily owing to decreased lean body mass suggested that LPS mobilizes amino acids from skeletal muscle to fuel ketogenesis. Indeed, levels of certain circulating amino acids, such as lysine and tyrosine, decreased and expression of catabolic enzymes increased in the liver after LPS administration. The LPS-treated mice also showed an accumulation of medium-chain and long-chain acylcarnitines in the plasma and liver, which indicates suppression of fatty acid oxidation. Together, these observations suggest that LPS induces a reprogramming of liver metabolism to favour catabolism of amino acids over fatty acids to support ketogenesis.

Plasma metabolomics also revealed an increase in triglycerides in LPS-treated mice housed at $30^{\circ} \mathrm{C}$. Plasma triglycerides are known to fuel cold-induced thermogenesis by brown adipose tissue. Accordingly, mice lacking the thermogenesis factor $U c p 1$ and housed at $22^{\circ} \mathrm{C}$ showed persistent hypothermia and decreased survival compared with those housed at $30^{\circ} \mathrm{C}$, suggesting that fatty acid-fuelled heat production by brown fat is necessary to exit from the hypometabolic-hypothermic state.

A host has two strategies to protect against pathogens resistance or tolerance. The authors asked whether competition for energy affects the defence strategy used. Mice infected with Listeria monocytogenes and housed at $22^{\circ} \mathrm{C}$ showed attenuated weight loss despite similar bacterial burden and inflammatory responses to mice housed at $30^{\circ} \mathrm{C}$, suggesting they were more tolerant to the infection. Similarly, despite similar inflammatory responses following Escherichia coli infection of both groups of mice, those housed at $22^{\circ} \mathrm{C}$ tolerated higher bacterial burdens, survived better and demonstrated an energetic trade-off with homeothermy. Failure to exit hypothermia owing to Ucp 1 deletion abrogated this tolerant phenotype.

On the basis that energyconserving hypometabolic states favour disease tolerance, the authors tested whether caloric restriction (every other day feeding) could improve survival in mice housed at $30^{\circ} \mathrm{C}$. Indeed, caloric restriction resulted in energy conservation on both fasting and fed days and was associated with a faster recovery from infection-induced weight loss and a significant survival advantage.

In summary, the authors show that competition for energy between immunity and homeothermy triggers entry into a hypometabolichypothermic state that enhances tissue tolerance. This strategy is also used in hibernating animals and thus may have evolved as a mechanism for tissue tolerance.

Lucy Bird

ORIGINAL ARTICLE Ganeshan, K. et al. Energetic trade-offs and hypometabolic states promote disease tolerance. Cell https://doi.org/10.1016/ j.cell.2019.01.050 (2019) 